\title{
Static and dynamic evaluations of the wettability of commercial vinyl polysiloxane impression materials for artificial saliva
}

\author{
Feng LUO'1, Guang HONG², Tong WANG ${ }^{1}$, Lingling JIA ${ }^{1}$, Jun-Yu CHEN ${ }^{1}$, Lai SUO ${ }^{1}$, Xi-Bo PEI ${ }^{1}$ and Qian-Bing WAN ${ }^{1}$ \\ ${ }^{1}$ State Key Laboratory of Oral Diseases, National Clinical Research Center for Oral Diseases, West China Hospital of Stomatology, Sichuan \\ University, No. 14, Section 3, South People's Road, Chengdu, China \\ ${ }^{2}$ Liaison Center for Innovative Dentistry, Graduate School of Dentistry, Tohoku University, Sendai 980-8575, Japan \\ Corresponding author, Qian-Bing WAN; E-mail: wanqianbing@126.com
}

\begin{abstract}
This study evaluated the wettability of commercial vinyl polysiloxane impression materials by measuring contact angles on horizontal and non-horizontal surfaces using artificial saliva. Three light bodies (Affinis [Affi], Silagum [Sila] and Variotime [Vario-LB]) and one extra light body (Viriotime [Vario-ELB]) were prepared with flat surfaces. Static and dynamic contact angles were measured using the sessile drop method as a function of time. Contact angle hysteresis was the value of advancing contact angle minus receding contact angle. The results indicated that all materials were classified as hydrophilic materials. Vario-ELB and Affi showed better initial wettability than the other two materials. Vario-ELB showed more hydrophilic properties and was more vulnerable to changes in the intraoral environment compared to Affi, Sila and Vario-LB. These results suggest that measuring the time-dependent dynamic contact angle on the inclined surface can provide effective information regarding the wettability of impression materials to evaluate their clinical performance.
\end{abstract}

Keywords: Vinyl polysiloxane, Wettability, Artificial saliva, Contact angle hysteresis

\section{INTRODUCTION}

Addition-type vinyl polysiloxane (VPS) impression materials are widely used in dentistry, because of their favorable physical properties, handling characteristics and dimensional stability ${ }^{1,2)}$. Despite the many advantages of VPS as a material that accurately replicates intramural tissue, its poor wettability, which is caused by its hydrophobic polymeric structures, is recognized as a significant limitation ${ }^{3)}$. The term "wettability" refers to the ease with which liquid spreads across a solid surface or, more specifically, how the liquid adheres to a solid surface ${ }^{4}$. Wettability is an important feature for a void-free cast to accurately reproduce oral tissue. When taking dental impression, poor wettability will cause problems during the process of creating a detailed impression of the intraoral tissues and the process of producing a detailed replica on the VPS ${ }^{5}$. However, to ensure clinical success, manufacturers continually try to make the inherently hydrophobic siloxane-based materials more hydrophilic ${ }^{6}$. Several techniques, such as involving incorporation of a surface tension eraser or wetting conditioner surfactant ${ }^{7}$, treatment with radiofrequency glow-discharge or plasma ${ }^{2,8)}$, have been reportedly use to modify the surface properties of VPS. Thus, a precise investigation of the wettability of VPS impression materials is important to evaluate their clinical performance.

An effective method for observing wetting is to measure the contact angle formed between a drop of liquid and the material's surface ${ }^{9}$. Contact angles, including the static contact angle (SCA) and dynamic contact angle (DCA, contains the advancing contact angle $[\mathrm{ACA}]$ and receding contact angle [RCA]), are widely used to quantitatively characterize the hydrophilicity, as well as the hydrophobicity, of a material. Furthermore, because of the fluctuation of drop caused by inertial effects during any initial period of the drop/material contact $(0-3 \mathrm{~s})$, the SCA measured at a contact time of approximately three seconds is referred to as the initial contact angle (ICA) to investigate the material's intrinsic properties ${ }^{6,10)}$. As previously described, a material exhibiting contact angle value higher than $90^{\circ}$ is considered to be hydrophobic, while a value smaller than $90^{\circ}$ is recognized as hydrophilic ${ }^{11}$. Furthermore, contact angle hysteresis (CAH), which is defined as the difference between the ACA and RCA, is also commonly used to study the chemical and physical heterogeneities of materials ${ }^{12)}$.

However, few studies have examined the contact angle of impression material on an inclined surface. Most previous studies have measured the SCA of set and unset impression materials on a horizontal surface $e^{2,13,14)}$. Moreover, some publications have measured the DCA using the Wilhelmy plate method, with the specimen suspended or immersed in wetting liquid ${ }^{4,10)}$. Although these methods are simple and effective, the obtained information is limited because these methods only determine the wettability on a horizontal or vertical surface. Conversely, the information obtained from measuring time-dependent DCA and $\mathrm{CAH}$ on an inclined surface is more clinically relevant. It is well known that when taking impressions or making a plaster cast, the surfaces of tooth structures, periodontal tissues and implants, particularly the areas of gingival sulcus and shoulder, are chambered or inclined, instead of being horizontal or vertical. Therefore, to evaluate the clinical performance of impression materials, it is necessary to 
provide effective information regarding the wettability of impression materials through more research of the wet properties of impression materials.

Currently, the sessile drop method is regarded as an appropriate approach to evaluate the hydrophilicity, as well as the hydrophobicity, of impression material ${ }^{15)}$. The SCA is determined for the three-phase contact line that remains stationary during the measurement. In addition, the sessile drop method can also measure advancing and receding contact angles by tilting the specimen ${ }^{16,17)}$. Schipper et al. have reported that artificial saliva is more clinically relevant compared to water ${ }^{18)}$. Therefore, using artificial saliva as a test liquid can satisfy the clinical requirements.

The purpose of this study was to evaluate the wettability of VPS impression materials by measuring time-dependent static and dynamic contact angles on horizontal and inclined surfaces with artificial saliva. In addition, the ICA and time-dependent $\mathrm{CAH}$ of four materials were also investigated. This study is the first to simultaneously evaluate the wettability of VPS impression materials on horizontal and non-horizontal surfaces. The null hypothesis was that the measuring method used in this study can effectively evaluate the wettability of impression materials.

\section{MATERIALS AND METHODS}

\section{Materials}

Three light body and one extra light body VPS impression materials were used in this study, as shown in Table 1. All materials were provided in cartridges with matching mixing tips and prepared with auto-mix dispenser, in compliance with the manufacturer's recommendations. The artificial saliva was made according to the report by Iijima et $a l .{ }^{19)}$. The artificial saliva was sterilized through autoclaving and stored at $4^{\circ} \mathrm{C}$.

\section{Specimen preparation}

A square-shaped stainless mold $\left(80 \times 10 \times 5 \mathrm{~mm}^{3}\right)$ was used to prepare the test specimens with flat surfaces. The VPS impression materials were injected into the mold and polymerized, according to the manufacturer's instructions. Three specimens from each material were selected for each experiment.

\section{Contact angle measurement}

Static and dynamic contact angles were measured using the sessile drop method with an OCA-20 contact angle analyzer (DataPhysics Instruments, Filderstadt, Germany), according to the Young-Laplace fitting method. A micro-syringe was used to ensure the uniformity of the artificial saliva droplet volume $(0.5$ $\mu \mathrm{L})$. The specimens were placed on a manual tilting stand, and the SCA was measured at a neutral tilting angle (Fig. 1a). Then, the tilting stand was tilted down $10^{\circ}$ to obtain a stable inclined surface. Subsequently, the advancing and receding contact angles were determined (Fig. 1b). CAH was observed as the value of the advancing minus the receding contact angles. Continuous images

Table 1 Information of the materials tested

\begin{tabular}{llcclc}
\hline \multicolumn{1}{c}{ Code } & Materials & Lot No. & $\begin{array}{c}\text { Setting time } \\
(\text { min: s })\end{array}$ & Manufacturer & Types \\
\hline Affi & Affinis & C16888 & $2: 00$ & $\begin{array}{l}\text { Coltène/Whaledent, Sankt Gallen } \\
\text { Area, Switzerland }\end{array}$ & Light body \\
Sila & Silagum & 661123 & $3: 30$ & DMG, Hamburg, Germany & Light body \\
Vario-LB & Variotime & 360240 & $2: 30$ & Heraeus, Hanau, Germany & Light body \\
Vario-ELB & Variotime & 360208 & $2: 30$ & Heraeus & Extra light body \\
\hline
\end{tabular}

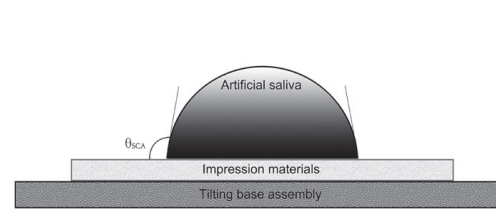

Static contact angle

(a)

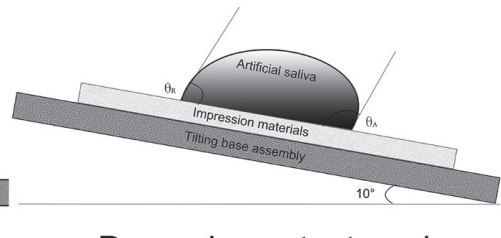

(b)

Fig. 1 A schematic of contact angle measurement using the sessile drop method with artificial saliva.

(a) The static contact angle was measured at a neutral tilting angle; (b) the advancing and receding contact angles were measured by tilting the specimen. The contact angle hysteresis was the value of the advancing minus the receding contact angles. 
were captured when an artificial saliva drop contacted the specimen surface. For the SCA, a total of $20 \mathrm{~s}$ was recorded. In addition, the ICA, referred to as the magnitude of the SCA taken at a contact time of $3 \mathrm{~s}$, was specifically analyzed and determined through the Ellipse and Young-Laplace fitting methods. For the DCA, a total of $15 \mathrm{~s}$ for the artificial saliva drop to contact the surface of specimen was recorded. The interval of each recording was $1.000 \pm 0.012 \mathrm{~s}$. Five replicates were made per formulation. All measurements were performed at room temperature, with a relative humidity of $40 \pm 2 \%$.

\section{Statistical analysis}

All SCA, ICA, ACA, RCA and CAH data were independently analyzed using one-way analysis of variance (ANOVA) and the Games-Howell post hoc test at a 5\% level of significance, using SPSS 17.0 software.

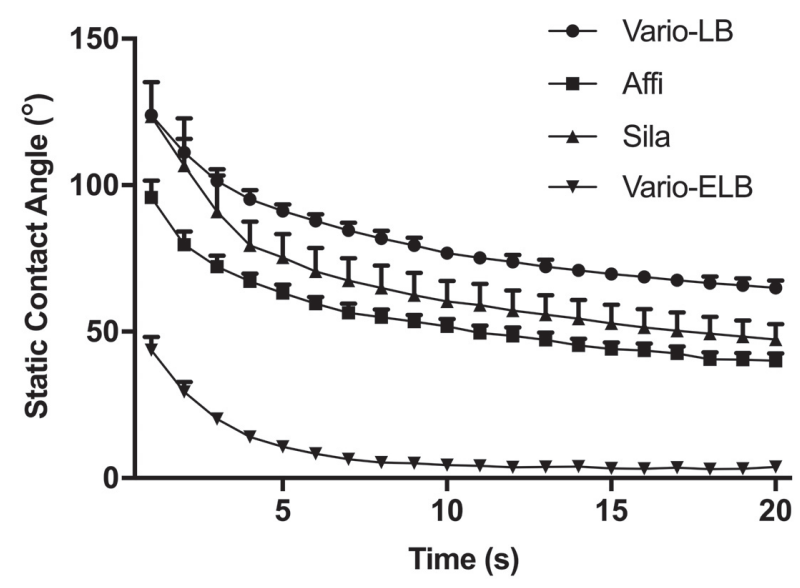

Fig. 2 The variations of SCA, according to the measurement time.

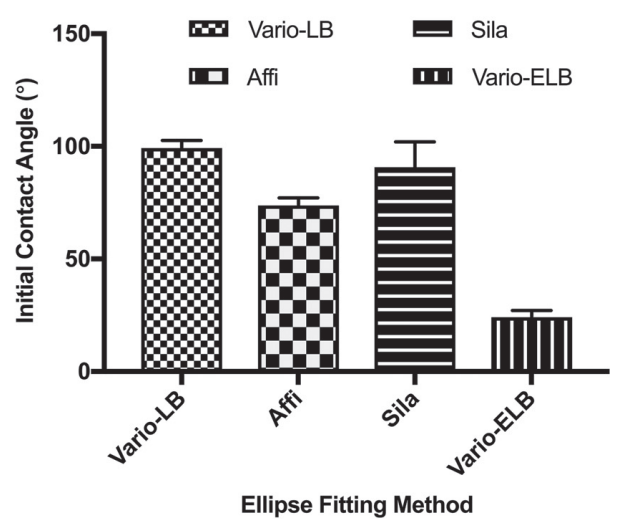

(a)

\section{RESULTS}

$S C A$

The SCA of each impression materials is shown in Fig. 2. Figure 3 provides representative images of the contact between the artificial saliva drops and specimen surfaces at different times (i.e., 1, 3, 8, 20 s). The SCA of all materials decreased significantly as time increased $(p<0.05)$. A rapid change in the SCA was recorded until 5 s. During the measurement period, Vario-ELB had significantly lower SCA values than the other three materials $(p<0.05)$. Vario-LB showed significantly higher SCA values than Affi $(p<0.05)$.

$I C A$

The average value (with standard deviation) for ICA is shown in Fig. 4. Similar results were shown for the

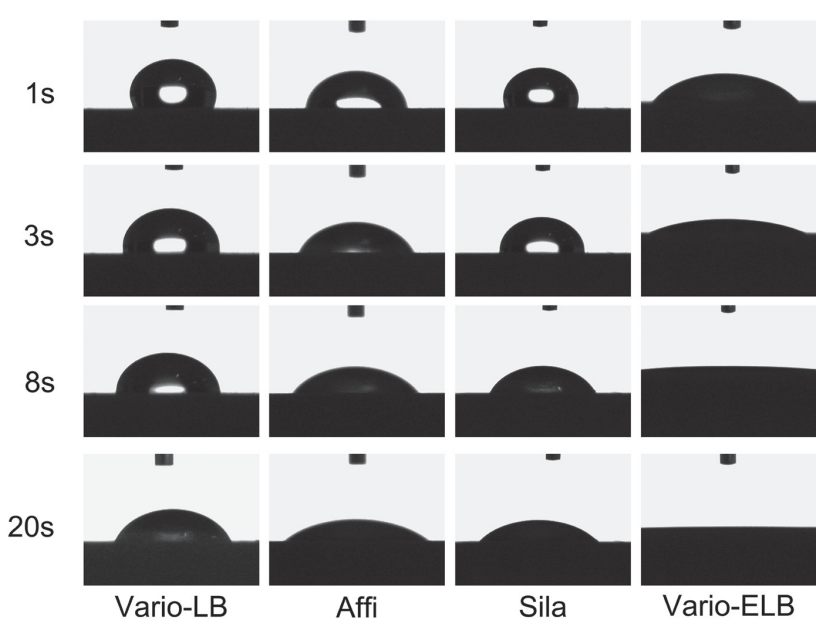

Fig. 3 Representative SCA images of contact between artificial saliva drops and specimen surfaces at different times (i.e., 1, 3, 8, $20 \mathrm{~s}$ ).

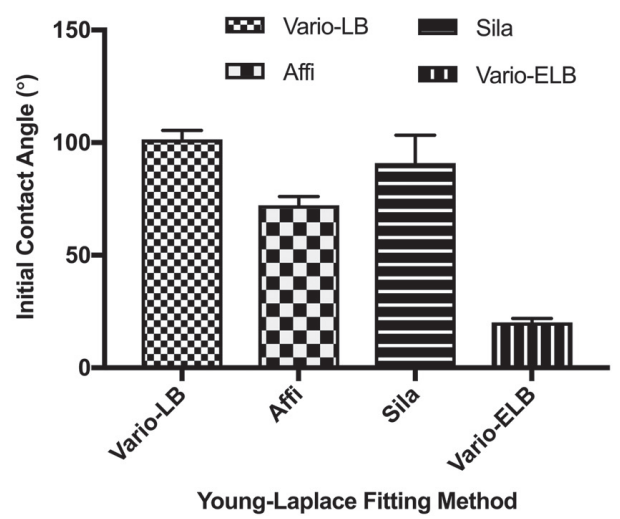

(b)

Fig. 4 The average values, with standard deviation, of the ICAs of Vario-ELB, Affi, Sila and Vario-LB.

(a) The ICA measured with the Ellipse fitting method; (b) The ICA measured with the Young-Laplace fitting method. 
Ellipse and Young-Laplace fitting methods. Vario-ELB demonstrated the lowest ICA values (Ellipse fitting method: $24.13 \pm 3.09^{\circ}$ and Young-Laplace fitting method: $20.20 \pm 1.71^{\circ}$ ) compared with three light body impression materials $(p<0.05)$. Sila and Vario-LB showed the ICA values that were higher than $90^{\circ}$. In addition the ICA values for Vario-ELB and Affi were lower than $90^{\circ}$.

\section{$D C A$}

Figure 5 shows representative DCA images of contact between the artificial saliva drops and inclined surfaces at different times (i.e., 1, 3, 8, $15 \mathrm{~s}$ ). Variations in the advancing and receding contact angles, according to time of measurement, are shown in Fig. 6. All tested VPS impression materials showed characteristic changes in wet properties with time. After a few seconds from the first measurement, the ACA values (Fig. 6a) decreased to lower than $90^{\circ}$ for three light body materials, which was consistent with the SCA results. Throughout the experimental period, the ACA of Vario-ELB was significantly lower than the other three materials

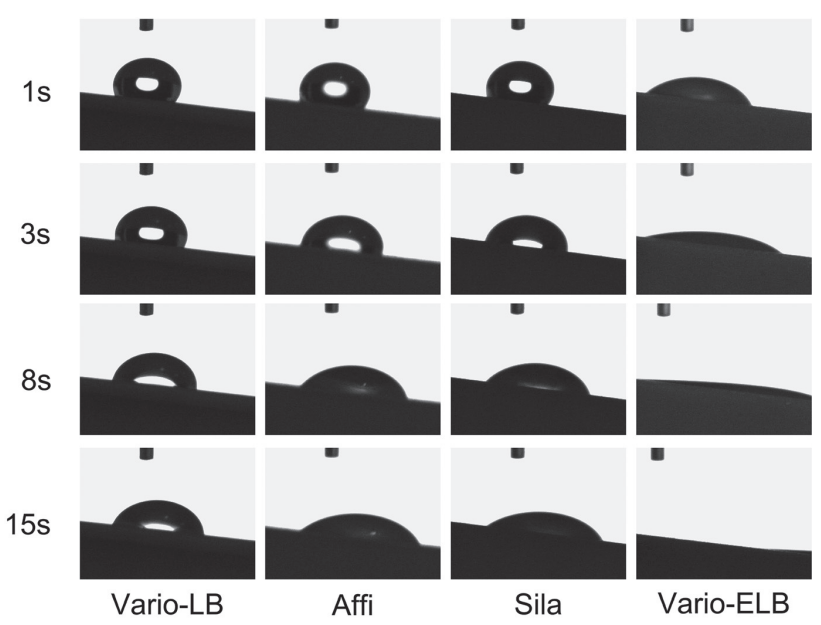

Fig. 5 Representative DCA images of contact between the artificial saliva drop and inclined surface at different times (i.e., 1, 3, 8, 15 s).

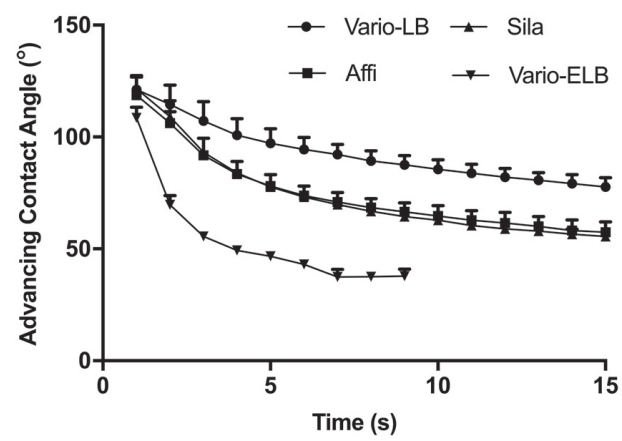

(a) $(p<0.05)$. The RCA values (Fig. 6b) of Affi, Sila and VarioLB were $44.59 \pm 3.91^{\circ}, 41.63 \pm 2.14^{\circ}$ and $67.13 \pm 4.47^{\circ}$, respectively, at a contact time of $15 \mathrm{~s}$. For Vario-ELB, the RCA values was $14.00 \pm 0.14^{\circ}$ at a contact time of $8 \mathrm{~s}$, which was significantly lower than the other materials' $(p<0.05)$. The ACA and RCA of Vario-ELB were not recorded after $8 \mathrm{~s}$ because the drop spread down along the inclined specimen surface and could not be captured by the CCD video camera.

\section{$C A H$}

Figure 7 shows the CAH of four impression materials under the dynamic condition as a function of time. Note that the $\mathrm{CAH}$ for all materials increased with time, in contrast to the SCA and DCA results (Figs. 2, 6). The Vario-ELB showed the highest degree of hysteresis compared to the other materials. The CAH of all materials increased as time increased. A rapid change in $\mathrm{CAH}$ was recorded for Vario-ELB. The CAH values increased from $5.61 \pm 0.54^{\circ}$ to $12.89 \pm 0.76^{\circ}$ for Affi and from $5.55 \pm 0.21^{\circ}$ to $13.93 \pm 0.62^{\circ}$ for Sila, throughout the experimental period.

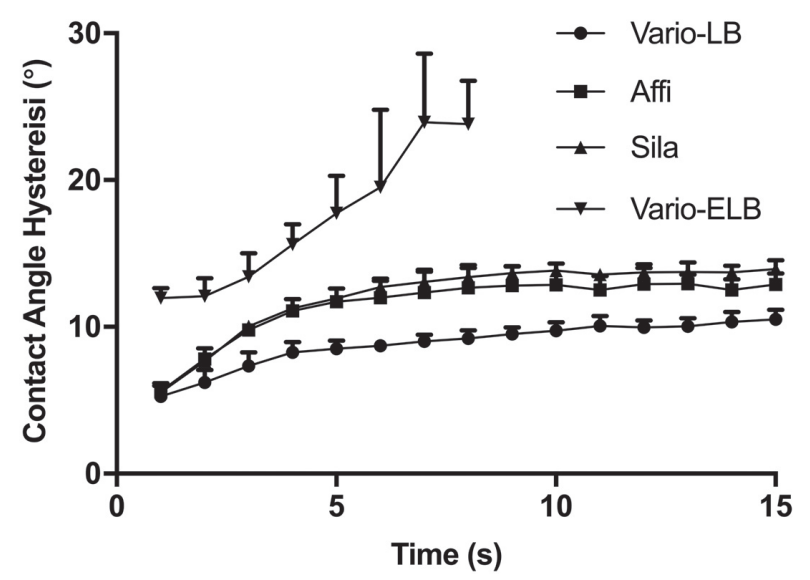

Fig. $7 \mathrm{CAH}$ variations, according to the measurement time.

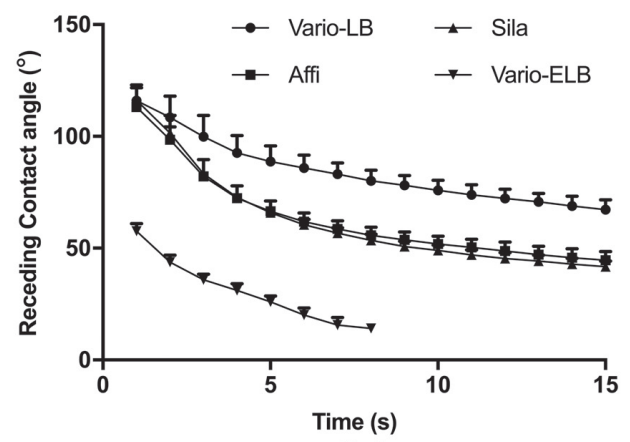

(b)

Fig. 6 DCA variations, according to the measurement time. (a) ACA and (b) RCA. 


\section{DISCUSSION}

The hypothesis that the measuring method used in this study is effective to evaluate the wettability of impression materials was proven to be true and accepted. VPS materials are recognized to be inherently hydrophobic, which can be explained by the materials' chemical structure, with hydrophobic, aliphatic hydrocarbon groups surrounding the siloxane bond ${ }^{10,20)}$. Hence, a surfactant is added into the VPS materials to produce hydrophilic or even ultra-hydrophilic impression materials. The surface concentration of surfactant on the silicone impression material has also been referred to as a decisive factor in determining surface hydrophilicity ${ }^{21}$. The surfactant used to set VPS impression materials can lower the contact angle and reduce voids in the recovered cast. If a surfactant is more hydrophobic, the obtained dispersion size is smaller. The surfactant in VPS materials theoretically reduces a contact angle in two manners ${ }^{13)}$. In the first theory, wettability is increased as the surfactant moves to the VPS surface. In the second theory, the surfactant is released from the VPS surface and reduces the surface tension of the liquid. Therefore, the surfactant in the VPS impression materials must move to the surface in the allowed time to induce the hydrophilicity of the surface, which is why many studies have focused on the wettability of un-polymerized materials, and regarded it as more clinically relevant. However, it is impossible to measure the advancing and receding contact angles of un-polymerized impression materials with a tiling stand because the un-polymerized materials will move along the tilting stand. There are several reports about the methods used to improve the wettability of $\mathrm{VPS}^{6,22)}$. Kwon et al. have reported that the improvement of hydrophilicity of VPS is possible when using nonthermal atmospheric-pressure plasma jet (NTAPPJ) as a substitute for a surfactant ${ }^{2)}$. The authors supposed that the oxidation of the VPS surface and a decrease in the hydrocarbon explained why the hydrophilicity of VPS improved through NTAPPJ exposure. In this study, the wet properties of one extra light body and three light body VPS impression materials were studied based on the SCA, ICA, ACA, RCA and CAH measurements using artificial saliva. Using artificial saliva instead of water as the testing liquid is due to the different reactions to surfactants. As reported, unlike water, saliva includes dissolver proteins and salts and cannot dissolve the surfactant released from VPS materials easier than water $^{14)}$. Therefore, the contact angle obtained using artificial saliva is slightly higher compared to water. However, to obtain the effective information regarding the wettability of impression materials to evaluate their clinical performance, saliva is more suitable as a testing liquid. For this reason, we used artificial saliva as the testing liquid in this study.

First, we measured the SCA over time to investigate the hydrophilicity of four VPS impression materials in this study. SCA is a traditional factor to evaluate the wettability, and SCA was calculated in this study using the sessile drop method. From the results, the contact angle for artificial saliva of these materials was found to be significantly dependent on the contact time (Figs. 2, 3). During the observation time of $20 \mathrm{~s}$, the contact angle remained small over time $\left(<90^{\circ}\right)$. We supposed that the decline of the SCA values might be caused by evaporation. To understand the characteristics of the materials, it was important to notice the initial hydrophilicity and the decline rates. The initial hydrophilicity was associated with the materials intrinsic properties ${ }^{2}$. In this study, the ICA values of four materials were measured using the Ellipse and Young-Laplace fitting methods to decrease the error and improve the measure precision (Figs. 2, 3). The ICA values of Affi and Vario-ELB were lower than $90^{\circ}$, suggesting that they have good initial hydrophilicity. In contrast, the relatively higher ICA values of Sila and Vario-LB $\left(>90^{\circ}\right)$ might be caused by the hydrophobic backbone chemistry of siloxanes ${ }^{6,22}$. However, the re-orientation of unreachable surface groups or the slowness of the leaching of surfactant from material may explain why higher values of SCA of Affi, Sila and Vario-LB were found compared to those of VarioELB $^{6}$. Vario-ELB had a smaller contact angle at every time during the individual measurements compared to the other materials, which may be due to the smaller dispersion size and the modification of composition and concentration of the incorporated surfactant. The smaller dispersion size of the surfactant is more easily moved to the surface of the impression material, and it can increase the wettability of materials. In addition, the alteration in the composition and concentration of surfactant could influence the wettability of impression materials $^{14,21)}$.

Measuring the SCA is a common method of studying the wet properties of materials. However, the SCA does not consider the relative movement of the various interfaces and cannot sufficiently describe the hydrophilicity of materials. The DCA, due to the effect of the velocity of the liquid-solid-vapor line, is more clinically relevant to characterize the hydrophilic/ hydrophobic phenomenon ${ }^{23)}$. Furthermore, CAH is usually considered to be an extensive property and it can evaluate the stickiness of liquid to material surfaces ${ }^{24)}$. $\mathrm{CAH}$ is recognized as an intrinsic parameter to describe the liquid-solid interactions ${ }^{25}$. The DCA and CAH can provide an indication of a material's wettability when the surface is non-horizontal and hydrophobic or hydrophilic groups are exposed. Therefore, the ADA, RDA and CAH were measured using the tilt stand method, and a fixed inclination angle was used for these materials to avoid differences in the conditions in this study ${ }^{26)}$.

The ACA and RCA values of four impression materials corresponded to the SCA results, which indicated a similar decreasing trend. The results showed that the wettability of impression materials measured using the SCA and DCA were closely correlated. However, note that the SCA of Affi was lower than that of Sila for all drop/material contact periods, while the ACA and RCA of Affi were higher than those of Sila after contact times of $5 \mathrm{~s}$ and $6 \mathrm{~s}$, respectively. We supposed 
that these results might be caused by the different released rates and the decreasing surface tension rates for the added surfactants in these two impression materials ${ }^{27}$. After the first few seconds, the ACA values decreased to lower than $90^{\circ}$ for all materials. In addition, at the end of testing, the RCA values of the four tested materials remained small $\left(<90^{\circ}\right)$. Thus, according to the SCA and DCA results, these four impression materials can be classified as 'hydrophilic'. Moreover, Vario-ELB demonstrated a lower contact angle and decreased faster compared to the other three materials. This phenomenon may due to the migration of lower molecular weight species from the bulk to the surface ${ }^{28)}$.

Hysteresis occurs due to the reorientation of the functional groups at the surface of the material ${ }^{29)}$. The evolution of hysteresis is related to the physical surface properties and liquid surface energy on a structured pattern $^{30)}$. In this study, the four materials showed relatively low degrees of hysteresis. At the end of testing, the hystereses of Vario-ELB, Sila, Affi and Vario-LB were equal to $23.80 \pm 2.97^{\circ}, 13.93 \pm 3.11^{\circ}$, $12.89 \pm 1.76^{\circ}$ and $10.51 \pm 1.06^{\circ}$, respectively. Additionally, note that as the DCA values increased, the CAH values decreased. Furthermore, the increased ratio of $\mathrm{CAH}$ was also consistent with the decreased ratio of DCA. These results might be attributed to the effect of the surfactant on surface tension. As reported, the $\mathrm{CAH}$ was dependent upon the liquid surface tension, and $\mathrm{CAH}$ would increase dramatically when the surface tension decreased ${ }^{31)}$. Therefore, the higher CAH values of Vario-ELB might be due to the large amount and small size of the surfactant, which in turn led to the lower SCA and DCA. When the material was exposed to an artificial saliva drop, the hydrophilic surfactant groups moved toward the surface, instead of the hydrophobic groups within the VPS, which made the surface relatively more hydrophilic. Moreover, the surfactant reduced the surface tension of the liquid in contact, combined with other factors (e.g., gravity) that induced the drop of artificial saliva moving fast along the inclined specimen surface ${ }^{22)}$. This explanation may also be the reason that the testing time of Vario-ELB was limited ( $8 \mathrm{~s})$. However, it is difficult to say that the relatively higher hysteresis of Vario-ELB is a significant advantage. When preparing the negative mold, a lower surface tension than that of the oral tissue is preferred. In contrast, the set impression material should have a high surface tension to ensure complete wetting by the casting material ${ }^{6}$. Furthermore, when the moisture condition of the oral and material surface exhibit minimal hysteresis, it is relatively unaffected or 'inert' to such changes in the local environment ${ }^{29)}$, and it is more beneficial to accurately replicate the intraoral tissue details. Therefore, for dental impression materials, a surface with a low CA (a high degree of wettability) and low level of hysteresis ('inert' to the local environment) would be advantageous.

The clinical significance of this study is that VarioELB showed the lowest SCA, ICA, ACA and ACA in four tested materials. These results suggest that the VarioELB has better hydrophilicity than Affi, Sila and Vario-
LB. However, Vario-ELB also showed higher hysteresis, meaning that this material is more vulnerable to changes in intraoral environments. Some researchers have reported that the surfactant did not change the surface properties of the VPS itself when reducing the surface tension of the liquid in contact ${ }^{22}$. For example, it was reported that the consistency, as well as the linear dimensional accuracy of surfactant-modified silicone impression materials, were not affected by surfactants used in their studies ${ }^{21)}$. Linkevicius et al. have also reported that there were no significant differences between the extra light and normal VPS materials and the stability of a single open tray impression coping $^{32)}$. Furthermore, the extra-light body impression demonstrated that it was able to record all finer details using the new 2-step impression injection technique, without compression ${ }^{33)}$. Thus, the manufactures must make an appropriate compromise to address the surface energy, contact angle and hysteresis. However, VarioLB showed the lowest hysteresis among the four tested materials. These results suggest that Vario-LB is more 'inert' to the intraoral environments in clinical applications.

Those materials were modified with surfactant, but the manufacturer does not disclose the composition and concentration of surfactant used in. Therefore, further work exploring surfactant type would be beneficial. In addition, further studies involving the release rates of surfactants, the reactions of surfactants with testing liquids and the interaction between the materials and the dental stone plaster are necessary to understand the mechanism of the surface wettability of VPS impression materials and to prove the usefulness of dynamic evaluation methods in evaluating the clinical performance of impression materials.

\section{CONCLUSIONS}

The results of this study are summarized as follows.

1. All tested impression materials showed characteristic time-dependent changes in their static and dynamic contact angles.

2. All materials were classified as hydrophilic impression materials.

3. Vario-ELB demonstrated more hydrophilic properties and was more vulnerable to intraoral environment changes compared to Affi, Sila and Vario-LB.

With the limitation of the current study, our research suggests that measuring the time-dependent DCA on an inclined surface can provide effective information concerning the wettability of impression materials to evaluate their clinical performance. Static and dynamic evaluation of the contact angle is an effective method of evaluating the wettability of impression materials.

\section{REFERENCES}

1) Levartovsky S, Levy G, Brosh T, Harel N, Ganor Y, Pilo R. Dimensional stability of polyvinyl siloxane impression 
material reproducing the sulcular area. Dent Mater J 2013; 32: 25-31.

2) Kwon JS, Kim YH, Choi EH, Kim KN. Development of ultrahydrophilic and non-cytotoxic dental vinyl polysiloxane impression materials using a non-thermal atmosphericpressure plasma jet. J Phys D Appl Phys 2013; 46: 195201.

3) Basapogu S, Pilla A, Pathipaka S. Dimensional accuracy of hydrophilic and hydrophobic VPS impression materials using different impression techniques: An in vitro study. J Clin Diagn Res 2016; 10: 56-59.

4) Lad PP, Gurjar M, Gunda S, Gurjar V, Rao NK. The effect of disinfectants and a surface wetting agent on the wettability of elastomeric impression materials: An in vitro study. J Int Oral Health 2015; 7: 80.

5) Erkut S, Can G. Effects of glow-discharge and surfactant treatments on the wettability of vinyl polysiloxane impression materials. J Prosthet Dent 2005; 93: 356-363.

6) Hosseinpour D, Berg JC. The dynamic interaction of water with four dental impression materials during cure. J Prosthodont 2009; 18: 292-300.

7) Kurtulmus-Yilmaz S, Ozan O, Ozcelik TB, Yagiz A. Digital evaluation of the accuracy of impression techniques and materials in angulated implants. J Dent 2014; 42: 15511559 .

8) Surapaneni H, Attili S. Polyvinyl siloxanes in dentistry: An overview. Trends Biomater Artif Organs 2013; 27: 115-123.

9) Marmur A. Hydro-hygro-oleo-omni-phobic? Terminology of wettability classification. Soft Matter 2012; 8: 6867-6870.

10) Rupp F, Axmann D, Jacobi A, Groten M, Geis-Gerstorfer J. Hydrophilicity of elastomeric non-aqueous impression materials during setting. Dent Mater 2005; 21: 94-102.

11) Cwikel D, Zhao Q, Liu C, Su X, Marmur A. Comparing contact angle measurements and surface tension assessments of solid surfaces. Langmuir 2010; 26: 15289-15294.

12) Michalakis KX, Bakopoulou A, Hirayama H, Garefis DP, Garefis PD. Pre-and post-set hydrophilicity of elastomeric impression materials. J Prosthodont 2007; 16: 238-248.

13) Shetty S, Kamat G, Shetty R. Wettability changes in polyether impression materials subjected to immersion disinfection. Dent Res J 2013; 10: 539.

14) Menees TS, Radhakrishnan R, Ramp LC, Burgess JO, Lawson NC. Contact angle of unset elastomeric impression materials. J Prosthet Dent 2015; 114: 536-542.

15) Rupp F, Geis-Gerstorfer J. Hydrophilicity of unset and set elastomeric impression materials. Int J Prosthodont 2010; 23 : $552-554$.

16) Hong J, Kim YK, Kang KH, Oh JM, Kang IS. Effects of drop size and viscosity on spreading dynamics in DC electrowetting. Langmuir 2013; 29: 9118-9125.

17) ElSherbini AI, Jacobi AM. Retention forces and contact angles for critical liquid drops on non-horizontal surfaces. J Colloid Interface Sci 2006; 299: 841-849.

18) Schipper RG, Silletti E, Vingerhoeds MH. Saliva as research material: biochemical, physicochemical and practical aspects. Arch Oral Biol 2007; 52: 1114-1135.

19) Iijima M, Hashimoto M, Kohda N, Nakagaki S, Muguruma
T, Endo K, Mizoguchi I. Crystal growth on bioactive glass sputter-coated alumina in artificial saliva. Dent Mater J 2013; 32: 775-780.

20) Petrie CS, Walker MP, O'Mahony AM, Spencer P. Dimensional accuracy and surface detail reproduction of two hydrophilic vinyl polysiloxane impression materials tested under dry, moist, and wet conditions. J Prosthet Dent 2003; 90: 365372.

21) Oh YI, Lee DY, Hwang SY, Kim KN, Kim KM. Effect of non ionic surfactants on surface properties of hydrophilic polyvinyl siloxane impression materials. Colloids Surf A Physicochem Eng Asp 2003; 229: 9-17.

22) Balkenhol M, Haunschild S, Lochnit G, Wöstmann B Surfactant release from hydrophilized vinylpolysiloxanes. J Dent Res 2009; 88: 668-672.

23) Peng H, Birkett GR, Nguyen AV. The impact of line tension on the contact angle of nanodroplets. Mol Simul 2014; 40: 934-941.

24) Wu J, Xia J, Lei W, Wang BP. Advanced understanding of stickiness on superhydrophobic surfaces. Sci Rep 2013; 3: 3268.

25) Wang FC, Zhao YP. Contact angle hysteresis at the nanoscale: a molecular dynamics simulation study. Colloid Polym Sci 2013; 291: 307-315.

26) Raufaste C, Cox S. Deformation of a free interface pierced by a tilted cylinder: variation of the contact angle. Colloids Surf A Physicochem Eng Asp 2013; 438: 126-131.

27) Zgura I, Beica T, Mitrofan IL, Mateias CG, Pirvu D, Patrascu, I. Assessment of the impression materials by investigation of the hydrophilicity. Dig J Nanomater Biostruct 2010; 5: 749755.

28) Pinto S, Alves P, Matos CM, Santos AC, Rodrigues LR, Teixeira JA, Gil MH. Poly (dimethyl siloxane) surface modification by low pressure plasma to improve its characteristics towards biomedical applications. Colloids Surf B Biointerfaces 2010; 81: $20-26$.

29) Menzies KL, Rogers R, Jones L. In vitro contact angle analysis and physical properties of blister pack solutions of daily disposable contact lenses. Eye Contact Lens 2010; 36: 10-18.

30) Eral HB, Oh JM. Contact angle hysteresis: a review of fundamentals and applications. Colloid Polym Sci 2013; 291: 247-260

31) Wong TS, Kang SH, Tang SK, Smythe EJ, Hatton BD, Grinthal A, Aizenberg J. Bioinspired self-repairing slippery surfaces with pressure-stable omniphobicity. Nature 2011; 477: 443-447.

32) Linkevicius T, Svediene O, Vindasiute E, Puisys A, Linkeviciene L. The influence of implant placement depth and impression material on the stability of an open tray impression coping. J Prosthet Dent 2012; 108: 238-243.

33) Varvara G, Murmura G, Sinjari B, Cardelli P, Caputi S. Evaluation of defects in surface detail for monophase, 2-phase, and 3-phase impression techniques: An in vitro study. J Prosthet Dent 2015; 113: 108-113. 